\title{
A unified method to predict diffuse and localized instabilities in sands
}

\author{
WaiChing Sun* \\ Mechanics of Materials, Sandia National Laboratories, Mail Stop 9912, 7011 East Avenue, Livermore, CA 94551, USA
}

(Received 6 October 2011; final version received 14 May 2012)

\begin{abstract}
A simplified method to analyse diffuse and localized bifurcations of sand under drained and undrained conditions is presented in this paper. This method utilizes results from bifurcation analysis and critical state plasticity theory to detect the onset of pure and dilatant shear band formation, static liquefaction and drained shear failures systematically. To capture the soil collapse observed in experiments, the instability state line concept originated by Chu, Lo and Lee in 1993 is adopted. Emphasis is given to examine how the presence of pore-fluid may facilitate or delay instability after yielding occurs. The predictions of instabilities are compared with experimental data from triaxial compression tests on Toyoura and Changi sands.
\end{abstract}

Keywords: bifurcations; static liquefaction; diffuse instability; shear band; instability state line

\section{Introduction}

The stability of fluid-infiltrated porous solids, such as sand, rock, concrete and bone, is governed by constitutive responses of the solids and the interactions among solid grains and porefluid. Bifurcation analyses conducted by Rudnicki and Rice (1975) and Rudnicki (2009) have revealed that substantial plastic deformation of a porous solid may lead to the development of inhomogeneous strain concentrated in a narrow zone as an alternative to homogeneous deformation. Recent advances in experimental techniques such as particle image velocimetry, however, have shown that shear bands are not the only bifurcation modes observable in the laboratory (Ikeda et al. 2006). Instead, a diffuse, convection-like barrel-shaped bifurcation mode that preceded the formation of shear bands was observed in soil. Despite the difference in kinematics features, Borja (2006a,b) has shown that both localized and diffuse instability can be predicted via bifurcation analysis, provided that the kinematic features of the deformation mode (diffuse and localized) are taken into account properly.

Prediction of both the onset and type of instability is critical for many geotechnical and geomechanical applications, such as slope stability analysis, ground improvement for liquefiable deposit and underground $\mathrm{CO}_{2}$ storage. For instance, Terzaghi (1943) estimates the bearing capability of foundations by assuming soil deposits behave as rigid blocks with localized failure surfaces. This assumption, however, may not be relevant if the soil deposit underneath the foundations fails in a diffusive mode under static liquefaction.
Predicting both the onset and types of failure at both the drained and undrained limits is not a trivial task, primarily because the rate-dependence and pore-pressure-gradientdependence introduced by the coupling between inelastic deformation and pore-fluid flow makes local, homogeneous analysis invalid. However, if the material of interest is at either the fully drained or undrained limit, then the presence of pore-fluid becomes only a volumetric constraint to porous solid, which legitimatizes the bifurcation analysis of instantaneous material properties at the material point level.

By taking full advantage of this simplification at drainage limits, we establish a simple and unified bifurcation analysis approach to assess stability and predict the instability modes of sands, without sacrificing the rigour of correct physics. Our goal here is to achieve a balance between simplicity and sophistication of concepts related to instabilities and bifurcations such that the analytical framework can be eventually useful for practising engineers.

The two major building blocks of this unified approach are the critical state plasticity model responsible for replicating constitutive responses of sand (Manzari and Dafalias 1997, Dafalias and Manzari 2004) and the bifurcation criteria detecting localized and diffuse types of instability modes from loss of uniqueness (Hill 1958, Rudnicki and Rice 1975, Raniecki 1979, Runesson et al. 1996, Rudnicki 2004, Borja 2006a,b, Darve et al. 2007, Andrade 2009, Rudnicki 2009). To simplify the analysis further, we assume that plastic yielding is primarily a frictional mechanism and that an increase in stress under a constant stress ratio is assumed to cause

*Email: wsun@sandia.gov

(C) 2013 Taylor \& Francis 
only elastic strain (Dafalias and Manzari 2004). As a result, any cap-surface yielding mechanism and the corresponding instability modes, such as compaction banding (Issen and Rudnicki 2000, Issen 2002, Rudnicki 2004) and cataclastic flow ((Borja 2006a, 2007), are not considered here. As a result of this assumption, the pressure-sensitive plasticity sand model developed by Dafalias and Manzari (2004) becomes our obvious choice. While not accounting for any cap-surface yielding behaviour, this model incorporates concepts from critical state soil mechanics (Schofield and Wroth 1968) to derive a kinematic hardening rule capable of replicating stress-strain responses remarkably well and yet maintains its simplicity by using the common two-invariant Drucker-Prager yield surface to predict the onset of yielding. The usage of the two-invariant Drucker-Prager yield surface makes it possible to describe incremental elasto-plastic responses and the instability criteria with only five independent scale parameters (i.e. bulk modulus $K$, shear modulus $G$, hardening modulus $K_{p}$, frictional coefficient $\mu$ and dilatancy factor $\beta$ ) and the plastic flow direction. Since the instability criteria are functions of material parameters that can all be measured and extracted in conventional experimental settings (e.g. triaxial, biaxial, simple shear, direct shear tests), we can predict diffuse and localized instabilities without further empirical interpretations.

As noted earlier, a more comprehensive stability analysis requires examination of the rate-dependent and non-local constitutive responses as well as the heterogeneous nature of the materials. These elements are beyond the scope of this paper. Nevertheless, simplified analyses of stability at drainage limits are useful for giving guidances for a fuller analysis and insights for conservative engineering designs.

In the following section, we provide an overview of limit conditions that trigger diffuse and localized instabilities under fully drained and undrained conditions. While the accuracy of the instability predictions strongly depends on the performance of the constitutive model (Issen 2002), these limit conditions are valid for any two-invariant model without cap surface.

\section{Drained responses}

The fully drained condition is achieved if the time scale of the loading is much larger than that of the pore-fluid flow. In this case, the influence of the pore-fluid flow on the stability of the solid skeleton is negligible. Hence, the constitutive relation is assumed to be rate-independent and local, without the need for introducing any poro-elasticity parameter.

\subsection{Onset of the drained diffuse collapse}

Assume that the material deforms homogeneously prior to reaching the instability points and that the elastic response is isotropic. Under axisymmetrical loading, the linearized relation between stress and total strain increments can be sufficiently expressed by a elasto-plastic tangential operator with scalar deviatoric and volumetric components, i.e.

$$
\underbrace{\left[\begin{array}{c}
\dot{p} \\
\dot{q}
\end{array}\right]}_{\dot{\sigma}}=\underbrace{\frac{1}{\chi}\left[\begin{array}{cc}
\chi K-K^{2} \mu \beta & -3 G K \beta \\
-3 G K \mu & 3 \chi G-9 G^{2}
\end{array}\right]}_{\boldsymbol{C}^{\mathrm{ep}}} \underbrace{\left[\begin{array}{c}
\dot{\boldsymbol{\epsilon}}_{v} \\
\dot{\boldsymbol{\epsilon}}_{\mathrm{s}}
\end{array}\right]}_{\dot{\boldsymbol{\epsilon}}}
$$

where $\chi=K_{p}+3 G+K \beta \mu$. The determinant of the elastoplastic operator $C^{\mathrm{ep}}$ can be expressed in terms of the hardening modulus $K_{p}$, the frictional coefficient $\mu$, the plastic dilatancy factor $\beta$, shear modulus $G$ and bulk modulus $K$ (Raniecki 1979, Willam 2002, Borja 2006a), i.e.

$$
\operatorname{det}\left(\boldsymbol{C}^{\mathrm{ep}}\right)=\frac{3 K G K_{p}}{\chi}
$$

where the eigenvalues of $C^{\text {ep }}$ are 0 and $3 G K(1+\beta \mu) / \chi$ when $K_{p}=0$. The non-trivial eigen-strain associated with the zero eigenvalue of the elasto-plastic tensor takes the form

$$
\llbracket \dot{\boldsymbol{\epsilon}} \rrbracket=\frac{1}{3} \beta \boldsymbol{I}+\sqrt{\frac{3}{2} \boldsymbol{n}}
$$

where $\boldsymbol{n}$ is the spectral direction of the deviatoric stress increment (same as $\boldsymbol{R}^{\prime}$ in Dafalias and Manzari 2004). The physical consequence of $\boldsymbol{C}^{\mathrm{ep}}$ having a zero eigenvalue is that the material will not experience extra stress if a strain perturbation linearly proportional to the eigen-strain in Equation (3) is applied. In other words, the material loses controllability at $K_{p}=0$ under the drained condition.

\subsection{Onset of shear banding}

Rudnicki and Rice (1975) and Rudnicki (2009) have shown that any constitutive responses simulated by a two-invariant plasticity model has localized bifurcation points at which heterogeneous deformation may replace the initially homogeneous deformation while maintaining a uniform traction field normal to the band. The instability mode is localized and corresponds to a rank-one eigen-strain (strain that is the dyadic product of two independent vectors) that leads to zero stress traction across a narrow zone, i.e.

$$
n \cdot C^{\mathrm{ep}}:(\boldsymbol{m} \otimes n)=n \cdot \llbracket \dot{\sigma}^{\prime} \rrbracket=\mathbf{0}
$$

where $\boldsymbol{n}$ is a unit vector normal to the planar band. A non-trivial solution for $\boldsymbol{m}$ is possible only if the drained acoustic tensor becomes singular, i.e.

$$
\operatorname{det}\left|n_{i} C_{i j k l}^{\mathrm{ep}} n_{l}\right|=0
$$

This critical condition that triggers strain localizations can be expressed as a function of the hardening modulus $H$, frictional coefficient $\mu$, plastic dilatancy $\beta$ and the principal deviatoric stress, i.e. 


$$
\frac{K_{p}}{G}=\frac{K(\beta-\mu)^{2}}{4 G+3 K}-\frac{K\left[3 \sqrt{6} N_{2}+(\beta+\mu)\right]^{2}}{4(G+3 K)}
$$

where the scalar $N_{2}$ is the ratio between the intermediate principal deviatoric stress and the Euclidean norm of the principal deviatoric stresses. For material that does not exhibit cap-type plastic yielding, the plane of localization is orthogonal to the direction of the principal intermediate stress (Rudnicki and Rice 1975, Perrin and Leblond 1993, Issen 2002, Rudnicki 2004, 2009).

Moreover, shear band formation precedes drained diffuse collapse (i.e. shear bands form in the hardening regime) if the following relation is satisfied:

$$
(\beta-\mu)^{2}-\frac{4 G+3 K}{4(G+3 K)}\left(3 \sqrt{6} N_{2}+3 \beta+\mu\right)^{2}>0
$$

Clearly, this is not possible if the flow rule is associative $(\beta=\mu)$.

\section{Undrained responses}

Under the undrained condition, pore-fluid remains trapped inside the pores during the loading cycle and hence constrains the volumetric deformation of the solid skeleton. This volumetric constraint may facilitate or delay instability. Since porefluid flow within pores is negligible, the constitutive response is approximately rate-independent and pore-pressure-gradientindependent (Rudnicki 2009). The constitutive responses of the solids are nevertheless affecting how pore pressure builds up, and vice versa.

Constitutive relations for undrained responses can be expressed in the same form as Equation (1) by replacing the drained plasticity parameters with their undrained counterparts if (i) the elastic response remains isotropic, i.e. there is no coupling between the elastic deviatoric and volumetric responses and (ii) the effective stress is in Terzaghi form $\left(\sigma_{i j}+p \delta_{i j}\right)$ (Terzaghi 1943) or in Biot form $\left(\sigma_{i j}+B p \delta_{i j}\right)($ Biot 1941) and (iii) the inelastic increment in the apparent void volume fraction is equal to the inelastic volume strain increment (Rudnicki 2009).

\subsection{Onset of static liquefaction}

The coupled macroscopic constitutive responses of a porous solid at the undrained limit can be described by the elastoplastic material parameters augmented with the poro-elasticity parameters to replicate the coupling between the solid skeleton and pore-fluid. The momentum and mass balance of the uniform undrained solid subjected to axisymmetric setting is (Nur and Byerlee 1971, Rice and Cleary 1976, Borja 2006b)

$$
\left[\begin{array}{c}
\dot{p} \\
\dot{q} \\
0
\end{array}\right]=\left[\begin{array}{ccc}
K-K^{2} \mu \beta / \chi & -3 G K \beta / \chi & -B \\
-3 G K \mu / \chi & 3 G-9 G^{2} / \chi & 0 \\
-B & 0 & -1 / M
\end{array}\right]\left[\begin{array}{c}
\dot{\epsilon}_{v} \\
\dot{\epsilon}_{\mathrm{s}} \\
\dot{p}^{f}
\end{array}\right]
$$

where $B$ is the Skempton pore pressure coefficient, $M$ is Biot's modulus and $\dot{p}^{f}$ is the rate of change of pore pressure, for which the following relations hold (Coussy 2004):

$$
B=1-\frac{K}{K_{\mathrm{s}}} ; \quad \frac{1}{M}=\frac{B-\phi}{K_{\mathrm{s}}}+\frac{\phi}{K_{\mathrm{f}}}
$$

where $\phi$ is the porosity; $K_{\mathrm{s}}$ and $K_{\mathrm{f}}$ are the bulk modulus of the solid grains that form the solid skeleton and pore-fluid trapped inside. $B$ is introduced to account for the influence of excess pore pressure on effective stress when both the solid skeleton and the solid grains are compressible (i.e. $K / K_{\mathrm{s}} \neq 0$ ). For soil, $B \approx 1$, but it can be as low as 0.5 for concrete or rock (Zienkiewicz et al. 1999). On the other hand, Biot's modulus $M$ is introduced to account for the additional volume stored by the compression of pore-fluid $\left(\phi \dot{p}^{f} / K_{\mathrm{f}}\right)$ and the compression of grains $\left[(B-\phi) \dot{p}^{f} / K_{\mathrm{s}}\right]$ due to pore pressure increase. Interested readers should refer to Zienkiewicz et al. (1999) and Coussy (2004) for the details.

Condensing Equation (8) leads to the following relation:

$$
\underbrace{\left[\begin{array}{c}
\dot{p} \\
\dot{q}
\end{array}\right]}_{\dot{\sigma}}=\frac{1}{\chi} \underbrace{\left[\begin{array}{cc}
\chi\left(K+B M^{2}\right)-K^{2} \mu \beta & -3 G K \beta \\
-3 G K \mu & 3 \chi G-9 G^{2}
\end{array}\right]}_{\boldsymbol{C}^{\text {und }}} \underbrace{\left[\begin{array}{c}
\dot{\epsilon}_{v} \\
\dot{\epsilon}_{\mathrm{s}}
\end{array}\right]}_{\dot{\boldsymbol{\epsilon}}}
$$

The undrained tangential constitutive response expressed in Equation (8) ceases to remain stable if $\boldsymbol{C}^{\text {und }}$ becomes singular. This happens if the hardening modulus satisfies the following relation:

$$
K_{p}^{\mathrm{liq}}=-\frac{B^{2} M}{B^{2} M+K} K \mu \beta
$$

This result is identical to the static liquefaction criterion in Andrade (2009) $\left(K_{p}=-K \mu \beta\right)$ if both constituents are incompressible. Under axisymmetric loading, the non-trivial strain increment can be expressed as a tensor whose volumetric and deviatoric components maintain the following relation:

$$
\llbracket \dot{\boldsymbol{\epsilon}} \rrbracket=\frac{1}{3} \frac{K \beta}{B^{2} M+K} \boldsymbol{I}+\sqrt{\frac{3}{2} \boldsymbol{n}}
$$

In cases where the solid grains and pore-fluid are nearly incompressible (compared to the porous solid), $M \rightarrow \infty, B=$ 1 and the eigen-strain becomes isochoric. This implies that the porous solid can maintain the same deviatoric stress if a nontrivial, pure shear strain perturbation is applied. In other words, the material loses controllability at $K_{p}=-B^{2} M K \mu \beta /\left(B^{2} M+\right.$ $K) \approx-K \mu \beta$ under undrained conditions. Since the shear strength is lost at this point, the material would behave like a frictional fluid and hence can be considered to be liquefied. 


\subsubsection{Onset of the pure shear band}

Following the procedures in Rudnicki and Rice (1975) augmented with the pore-fluid mass balance law, one can derive the undrained limiting hardening modulus, which reads

$$
\begin{aligned}
\frac{K_{p}^{\mathrm{sb}}}{G}= & \frac{\left(K^{\text {und }}-B M\right)^{2}(\beta-\mu)^{2}}{K^{\text {und }}\left(4 G+3 K^{\text {und }}\right)} \\
& -\frac{\left[3 \sqrt{6} K^{\text {und }} N_{2}+\left(K^{\text {und }}-B M\right)(\beta+\mu)\right]^{2}}{4 K^{\text {und }}\left(G+3 K^{\text {und }}\right)} \\
& -\frac{B^{2} M K \mu \beta}{G K^{\text {und }}}
\end{aligned}
$$

where $K^{\text {und }}=K+B^{2} M$ is the undrained bulk modulus of the porous media. If both the pore-fluid and the solid grains are incompressible such that $M \rightarrow \infty$ and $B=1$, then Equation (13) can be simplified into

$$
\frac{K_{p}^{\mathrm{sb}}}{G}=-\frac{9}{2} N_{2}^{2}-\frac{K \mu \beta}{G}
$$

where the band angle is always $45^{\circ}$ to the principal stress regardless of the adopted yield criterion (Runesson et al. 1996, Rudnicki 2009). By comparing Equation (14) with Equation (11), we predict that undrained shear banding in materials composed of incompressible constituents always occurs in the softening regime and after static liquefaction for both associative and non-associative flow rules.

\section{Critical state plasticity model for sands}

We incorporate the instability state line concept (Chu et al. 1993, Lade 1993, Chu et al. 2003) into a critical state plasticity model. The framework of the critical state plasticity theory used here was developed by Manzari and Dafalias (1997) and Dafalias and Manzari (2004) (referred to as the MD model hereinafter). This model incorporates the bounding surface model concepts to manipulate volumetric and deviatoric hardening such that the simulated elasto-plastic constitutive response is consistent with critical state theory (Schofield and Wroth 1968). Interested readers should refer to the original paper cited above for details.

The MD model includes an empirical elasticity model that reads

$$
\dot{\boldsymbol{\epsilon}}_{i j}=\frac{1}{3 K(e, p)} \dot{p} \delta_{i j}+\frac{1}{2 G(e, p)} \dot{s}_{i j}
$$

where $s_{i j}=\sigma_{i j}-p / 3 \delta_{i j}$ is the deviatoric part of the Cauchy stress. The bulk modulus $K(e, p)$ and the shear modulus $G(e$, $p$ ) are functions of current mean stress $p$ and void ratio $e$, i.e.

$$
\begin{gathered}
K(e, p)=K_{o} \frac{(2.97-e)^{2}}{1+e}\left(\frac{p}{p_{\text {at }}}\right)^{1 / 2} ; \\
G(e, p)=G_{o} \frac{(2.97-e)^{2}}{1+e}\left(\frac{p}{p_{\text {at }}}\right)^{1 / 2}
\end{gathered}
$$

where $p_{\text {at }}$ denotes the atmospheric pressure. The elastic domain is enclosed by a purely kinematic hardening Drucker-Prager yield surface that reads

$$
f\left(s_{i j}, \alpha_{i j} p\right)=\left[\left(s_{i j}-\alpha_{i j}\right)\left(s_{j i}-\alpha_{j i}\right)\right]^{1 / 2}-\sqrt{\frac{2}{3}} m p=0
$$

where $m$ is a constant and $\alpha_{i j}$ is the back stress. The hardening rule of the material is modelled based on critical state theory (Schofield and Wroth 1968). In critical state theory, it is assumed that there exists a unique critical state under which materials experience large plastic shear without volume change. In the MD model, the critical state is reached if both the stress ratio (deviatoric over hydrostatic) $q / p$ and void ratio $e$ reach their corresponding critical values, i.e.

$$
\frac{q}{p}=\left(\frac{q}{p}\right)_{c}=M^{c} ; \quad e_{c}=e=e_{o}^{c}-\lambda_{c}\left(\frac{p}{p_{\mathrm{at}}}\right)^{\xi}
$$

where $M^{c}, e_{o}$ and $\xi$ are scalar parameters determined by interpolating the void ratio $e$, mean stress $p$ and deviatoric stress $q=\sqrt{3 / 2} s_{i j} s_{i j}$ at the observed critical state. To make the hardening and plastic dilatancy consistent with critical state theory, Dafalias and Manzari (2004) introduce two isotropic limit surfaces and use their distances from the critical state to manipulate the hardening modulus and plastic dilatancy. These two limit surfaces read as follows:

$$
q-M^{b} p=0 ; \quad M^{b}=M^{c} \exp \left[-n^{b}\left(e-e_{c}\right)\right]
$$

(hardening limit surface)

$$
q-M^{d} p=0 ; \quad M^{d}=M^{c} \exp \left[-n^{d}\left(e-e_{c}\right)\right]
$$

(dilatancy limit surface)

where $n^{d}$ and $n^{b}$ are scalar constants that control the size of the limit surfaces. They can be determined from the initial yielding points. The hardening modulus $K_{p}$ and plastic dilatancy factor $\beta$ are set to be continuous interpolation functions which depend on (i) the distance between limit and critical surfaces and (ii) other interpolation functions added to improve the accuracy of the MD model. In axisymmetrical settings, the hardening modulus $K_{p}$ and the dilatancy factor $\beta$ are

$$
\begin{gathered}
K_{p}=\frac{2 p h}{3}\left(M^{b}-\frac{q}{p}\right) ; \quad h=\frac{G_{o} h_{o}\left(1-c_{h} e\right)}{\sqrt{\frac{p}{p_{\mathrm{at}}}}\left|\frac{q}{p}-\left(\frac{q}{p}\right)_{\mathrm{in}}\right|} \\
\beta=A_{o}\left(1+<z_{i j} n_{j i}>\right)\left(M^{d}-\frac{q}{p}\right) ; \\
\dot{z}_{i j}=-c_{z}<-\epsilon_{v}^{p}>\left(z_{\max } n_{i j}+z_{i j}\right) \\
n_{i j}=\sqrt{\frac{3}{2}} \frac{1}{m p}\left(s_{i j}-\alpha_{i j}\right)
\end{gathered}
$$


where $n_{i j}$ is the deviatoric part of the yield surface gradient. $h$ and $A_{d}$ are functions introduced to improve interpolation of the hardening and dilatancy from experimental observations. $h_{o}$, $c_{h}, c_{z}$ and $z_{\max }$ are positive scalar material parameters obtained by trial and error. $\langle x\rangle$ is the Macaulay bracket, which is equal to $x$ if $x>0$ and 0 if $x<0 .(q / p)_{\text {in }}$ is the stress ratio at the beginning of the loading process. The tensor $z_{i j}$ is called the fabric-dilatancy tensor (Dafalias and Manzari 2004). It is introduced to take account of the content-normal orientation distribution changes during forward and reverse shearing.

Furthermore, the frictional coefficient $\mu$ can be obtained from the volumetric component of the yield surface, i.e.

$$
\mu=\sqrt{\frac{3}{2}} \frac{1}{m p^{2}}\left(s_{i j} s_{j i}-s_{i j} \alpha_{j i}\right)
$$

With the independent scale parameters (i.e. bulk modulus $K$, shear modulus $G$, hardening modulus $K_{p}$, frictional coefficient $\mu$ and dilatancy factor $\beta$ ) determined from the MD model, we have sufficient information to replicate the incremental linear tangential responses of the sands and assess the stability of the responses.

\subsection{Incorporating shear failure behaviour}

The constitutive relation developed so far is capable of replicating many important phenomenological characteristics of granular materials (Manzari and Dafalias 1997, Dafalias and Manzari 2004, Jefferies and Been 2006). However, the constitutive models described above predict a perfectly plastic response if $e=1 / c_{h}$, regardless of the amount of confining pressure to which the material is subjected. This is consistent with the fact that the instability line is defined by linking the top of the undrained paths, while assuming that it is independent of the confining pressure within a limited range (Lade and Pradel 1990, Chu et al. 1993, Imam et al. 2002, Chu et al. 2003, Wanatowski and Chu 2007a,b). However, if one wishes to model materials under a wide range of confining pressure with the same material parameter, then a phenomenological amendment can be made by rewriting $c_{h}$ as a function of effective hydrostatic pressure $p$. In that case, the expression $\left(1-c_{h}\right)$ in Equation (21) is replaced by the following relations:

$$
\begin{aligned}
K_{p} & =\frac{2 p h}{3}\left(M^{b}-\frac{q}{p}\right) ; \quad h=\frac{G_{o} h_{o}\left(1-c_{h}(p) e\right)}{\sqrt{\frac{p}{p_{\text {at }}}\left|\frac{q}{p}-\left(\frac{q}{p}\right)_{\text {in }}\right|}} ; \\
c_{h}(p) & =\frac{1}{e_{o}^{s}-\lambda\left(\frac{p}{p_{\text {at }}}\right)^{\xi}}
\end{aligned}
$$

where $e_{o}^{s}, s=\mathrm{L}, \mathrm{D}$ are the zero intercepts of the dilatant $\left(e_{o}^{\mathrm{D}}\right)$ and contractive $\left(e_{o}^{\mathrm{L}}\right)$ instability state lines in $e-p$ space. The instability state line is underneath the critical state line in the $e-p$ space if the material is dilatant and above the critical state line if the material is contractive.

\section{Numerical predictions}

In this section, we present fully drained and undrained numerical simulations at the material point level. The purpose of performing these simulations is twofold - (i) to compare instability predictions with experimental observations (ii) to analyse how the presence of pore-fluid affects stability. As shown in these numerical examples, the modified Manzari-Dafalias model is capable of replicating realistic constitutive responses of sand. This feature is important to ensure the accuracy of the instability predictions.

Both drained and undrained triaxial test simulations are conducted with the material parameters presented in Table 1 . The material parameters are calibrated with the drained triaxial compression tests conducted by Chu et al. (2003) (Changi sand) and undrained and drained triaxial compression tests by Verdugo and Ishihara (1996) (Toyoura sand).

\subsection{Example 1: Collapses in drained contractive Changi sand}

This simulation is conducted to replicate the contractive constitutive responses of the DR39 constant shear test reported in figure 12 of Chu et al. (2003). The drained triaxial test was conducted on Changi sand with initial void ratio $=0.95$. A $150 \mathrm{kPa}$ hydrostatic load is first applied on the Changi sand specimen, then the specimen is loaded in triaxial compression until the confining pressure reaches around $208 \mathrm{kPa}$ as shown in Figure 1a. Following this step, the confining pressure decreases under constant deviatoric stress. This decrease in confining pressure causes further axial strain in the specimen and makes the stress state closer to the critical state line as shown in Figures 1a-c. Our result shows that the simulated contractive response is in general in agreement with the experiments. In particular, our simulation is consistent with the observation that the loose, contractive specimen collapses at a stress state below the critical state line in $q-p$ space. As depicted in Figures 1d and 2, the contractive specimen exhibits perfectly plastic behaviour as both the plastic dilatancy

Table 1. Summary of material parameters used for numerical predictions

\begin{tabular}{llll}
\hline Symbol & Parameter & Changi sand & Toyoura sand \\
\hline$G_{o}$ & Shear coefficient & 150 & 125 \\
$\nu$ & Poisson ratio & 0.05 & 0.05 \\
$M^{c}$ & Critical stress ratio & 1.35 & 1.25 \\
$e_{o}^{c}$ & Critical void ratio parameter & 0.89 & 0.89 \\
$\lambda_{c}$ & Critical state parameter & 0.04 & 0.19 \\
$\xi$ & Critical state parameter & 0.4 & 0.7 \\
$e_{o}^{\mathrm{D}}$ & Instability state parameter & 0.72 & 0.85 \\
$e_{o}^{\mathrm{L}}$ & Instability state parameter & 0.98 & 1.08 \\
$h_{o}$ & Hardening parameter & 2.8 & 7.1 \\
$A_{o}$ & Dilatancy parameter & 0.94 & 0.704 \\
$z_{\mathrm{max}}$ & Dilatancy parameter & 4.0 & 4.0 \\
$c_{z}$ & Dilatancy parameter & 600 & 600 \\
$n^{b}$ & Limit surface size parameter & 1.1 & 1.1 \\
$n^{d}$ & Limit surface size parameter & 2.8 & 3.5 \\
\hline
\end{tabular}



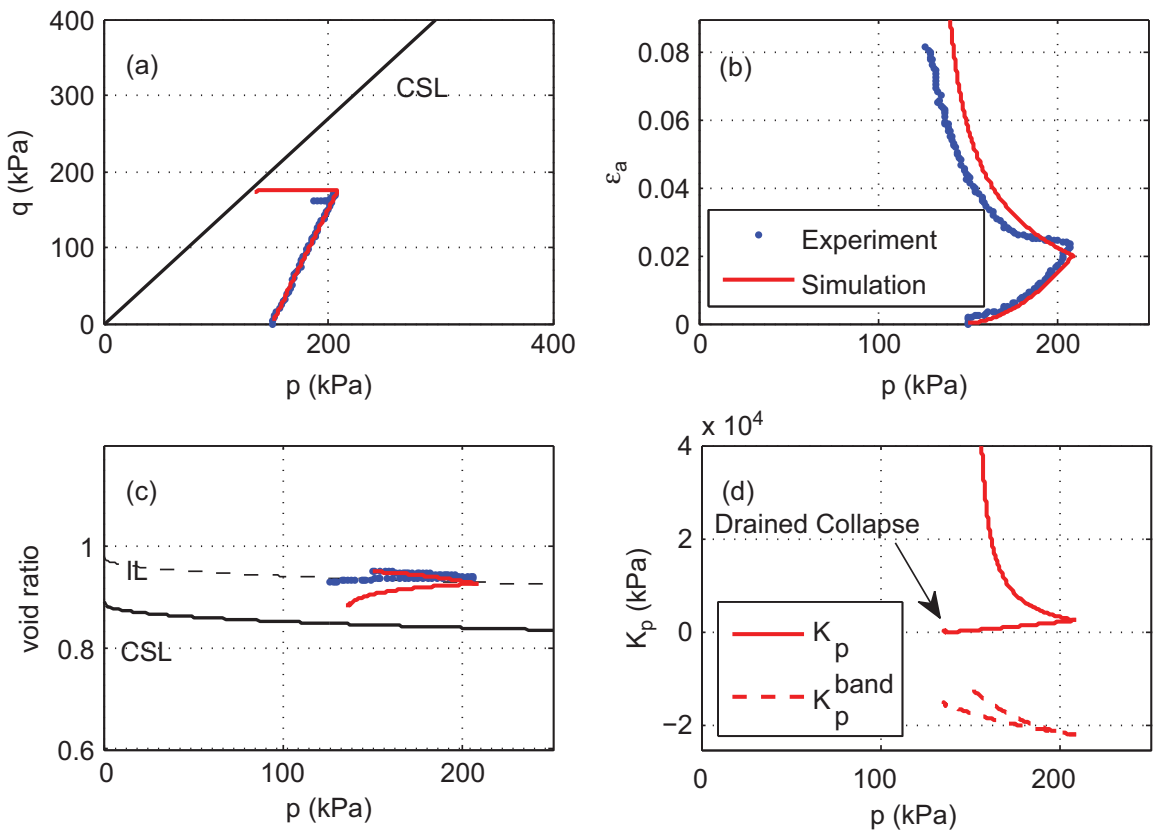

Figure 1. Numerical simulation of drained triaxial compression test on Changi sand with initial void ratio $=0.95$ (red): (a) simulated deviatoric stress, (b) axial strain, (c) void ratio, and (d) generalized hardening modulus versus effective hydrostatic pressure.

and the hardening modulus reduce to zero at $q / p=M^{b}=M^{d}$ (see Equations 21 and 22). As a result, a significant amount of shear strain develops without plastic dilatancy and thus leads to the collapse of the specimen. This unstable response replicated by the MD model is consistent with the observed rapid increase in axial strain of the specimen occurring before the stress passes through the critical state line as depicted in figure 7 of Chu et al. (2003). To quantify the asymptotically worst case of how much strain may develop in proportion to small changes in stress, we compute the condition number of the tangential tensor $\boldsymbol{C}^{\mathrm{ep}}$, which is defined as the ratio between the largest singular value and the smallest singular value

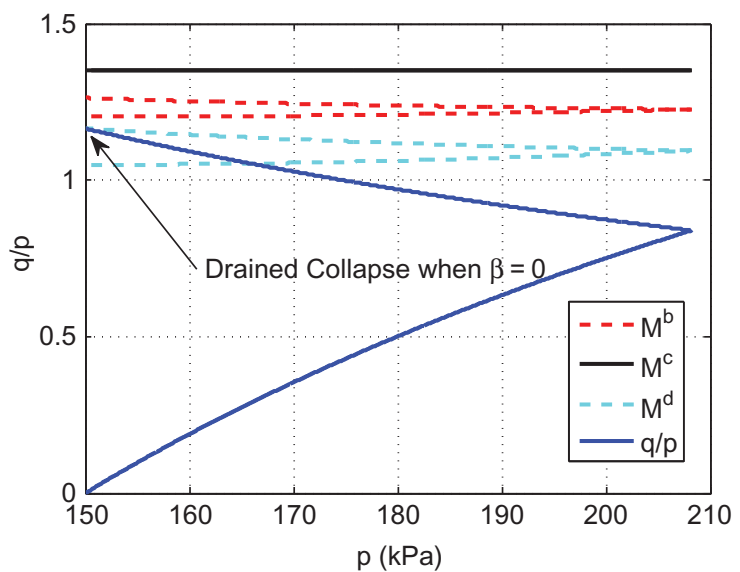

Figure 2. The stress ratio $q / p$ and the sizes of the hardening $\left(M^{b}\right)$ and dilatancy $\left(M^{d}\right)$ limit surfaces versus the effective hydrostatic pressure of the contractive Changi sand.
(Horn and Johnson 1985). A large condition number is an indication that the constitutive response of the specimen becomes unstable with respect to perturbation. A salient observation shown in Figure 3 is that the condition number of the tangent stiffness tensor actually increases much more rapidly when the generalized hardening modulus $K_{p}$ is approaching zero. Due to the deterioration in stability signalled by the increased condition number, a small stress perturbation might cause severe strain accumulation if the specimen is close to the instability state line. Since the hardening modulus remains close to zero in between the instability state line and the critical state line, this allows significant axial strain to develop, even though the

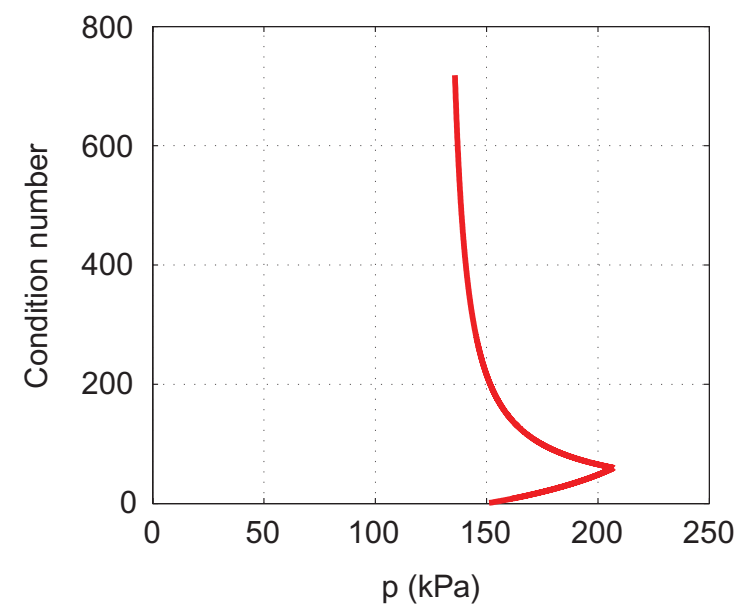

Figure 3. Condition number of $\boldsymbol{C}^{\mathrm{ep}}$ versus effective hydrostatic pressure of the contractive Changi sand. 


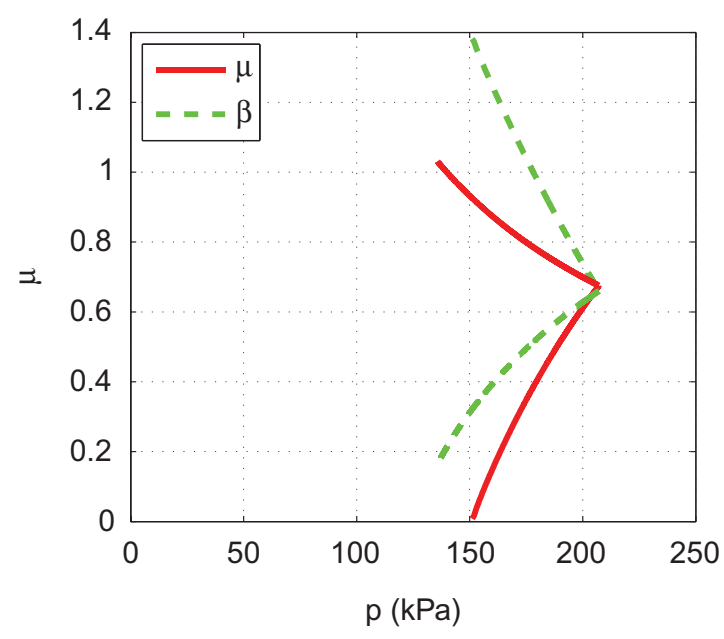

Figure 4. Plastic dilatancy $\beta$ and frictional coefficient $\mu$ versus effective hydrostatic pressure of the contractive Changi sand.

effective stress change is small. In this case, drained collapse may occur at the instability state line without a shear band being formed, as shown in Figure 1d.

The MD model predicts that both the plastic dilatancy $\beta$ and the frictional coefficient $\mu$ are positive after yielding, thus suggesting a contractive plastic behaviour as shown in Figure 4.

\subsection{Example 2: Formation of shear band in drained dilatant Changi sand}

This simulation is conducted to replicate the dilatant constitutive responses of the DR39 constant shear test reported in figure 12 of Chu et al. (2003). A triaxial load is applied under $150 \mathrm{kPa}$ confining pressure on Changi sand with a 0.657 initial

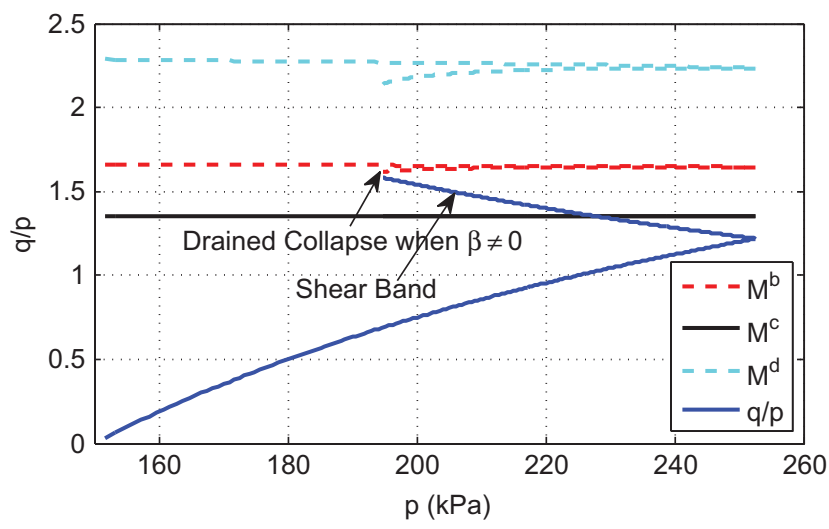

Figure 6. The stress ratio $q / p$ and the sizes of the hardening $\left(M^{b}\right)$ and dilatancy $\left(M^{d}\right)$ limit surfaces versus effective hydrostatic pressure of the dilative Changi sand.

void ratio. Figure 5a depicts the stress path. The specimen is first sheared until the deviatoric stress reaches $300 \mathrm{kPa}$. Then the deviatoric stress remains constant while the hydrostatic stress is decreasing. Our result shows that the simulated dilatant response is in general in agreement with the experiments.

By monitoring the generalized hardening modulus and its limit value for shear banding, we found that shear bands may form in the hardening regime when the effective hydrostatic pressure of the dilatant specimen is decreasing under constant deviatoric stress. The generalized hardening modulus $K_{p}$ remains positive but its magnitude decreases as the axial strain develops. This phenomenon is captured in the MD model by shortening the distance between the current stress and the instability line (denoted as IL in Figure 5c) and the distance between the current stress and the isotropic hardening limit surface as illustrated in Figure 6.
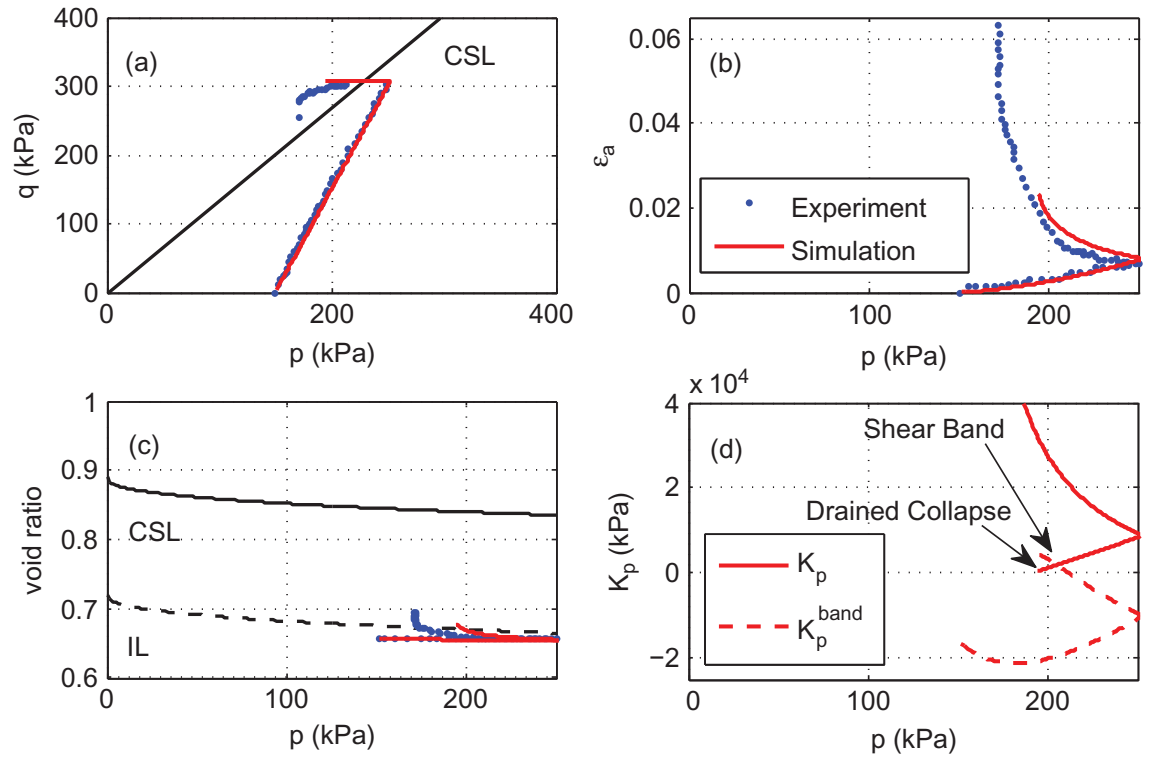

Figure 5. Numerical simulation of drained triaxial compression test on Changi sand with initial void ratio $=0.657$ (red): (a) simulated deviatoric stress, (b) axial strain, (c) void ratio, and (d) generalized hardening modulus versus effective hydrostatic pressure are plotted (red line) and compare with experimental observations (blue dots). 


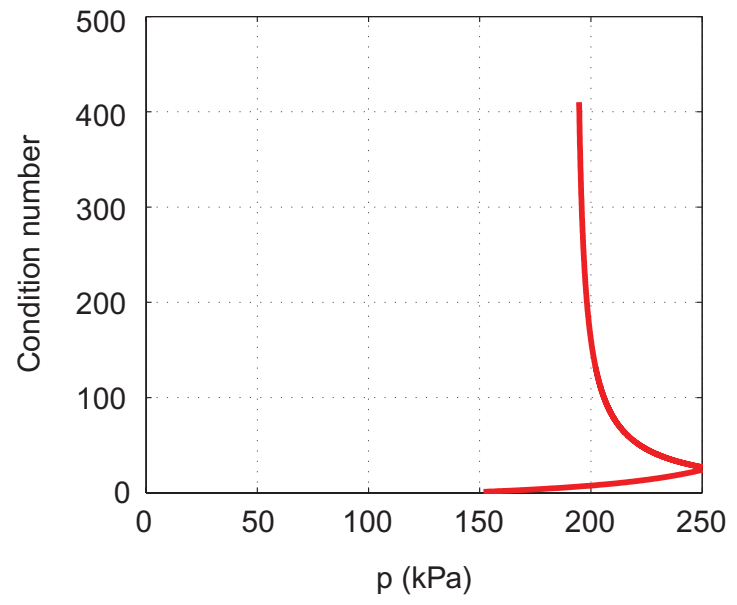

Figure 7. Condition number of $\boldsymbol{C}^{\mathrm{ep}}$ versus effective hydrostatic pressure of the dilatant Changi sand.

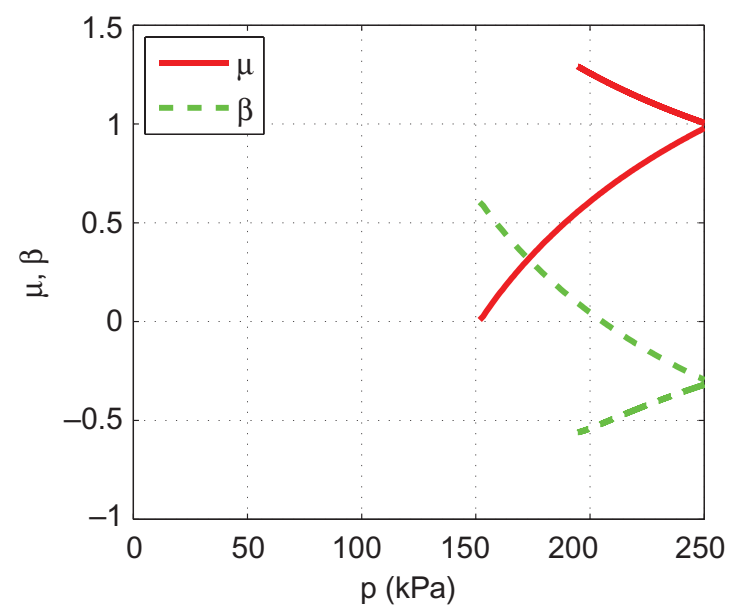

Figure 8. Plastic dilatancy $\beta$ and frictional coefficient $\mu$ versus effective hydrostatic pressure of the dilative Changi sand.

At the onset of shear band formation, the material exhibits plastic dilatancy (because $q / p<M^{d}$ ). Hence, the strain localization zone may exhibit both shear and dilatancy.

Following the onset of shear banding, the modified MD model predicts that the material would become very sensitive to perturbation as the response becomes more and more close to perfectly plastic as depicted in Figures $5 \mathrm{~b}$ and $5 \mathrm{~d}$. Notice that the dense, dilatant specimen collapses at a stress state above the critical state line in $q-p$ space when the current stress reaches the hardening limit surface, i.e. $q / p=M^{b}$ as depicted in Figure 6. This prediction is consistent with the observed rapid increase in axial strain of the specimen occurring after the stress passes through the critical state line described in figure 8 of Chu et al. (2003). To assess the stability of the constitutive response, we compute the condition number of $\boldsymbol{C}^{\mathrm{ep}}$, which is shown in Figure 7. By comparing the condition number of the loose and dense specimen at the instability state line, we found that the condition number of $\boldsymbol{C}^{\text {ep }}$ of the dense specimen is approximately one order smaller than that of the loose sand specimen, thus suggesting that the constitutive response of the dense Changi sand in Example 2 is more stable than the loose Changi sand counterpart in Example 1 when both are at the instability state line. Figure 8 shows the evolution of plastic dilatancy and frictional coefficient predicted by the MD model during the drained triaxial compression test. The plastic dilatancy is found to be negative after the onset of shear bands predicted via Equation (6), thus suggesting the formation of a dilatant shear band.

\subsection{Example 3: Static liquefaction of undrained Toyoura sand in loose state}

Three monotonic undrained triaxial compression tests on loose Toyoura sand are simulated and compared with the experiments conducted by Verdugo and Ishihara (1996). Figures 9a and 9b compare the simulated undrained constitutive response with the experiments. As demonstrated in Figure 9, the simulated constitutive responses are in good agreement with the experiments. Moreover, we found that softening only occurs if the loose Toyoura sand is first consolidated under sufficient confining pressure. In the monotonic undrained triaxial compression test with confining pressure $p^{\prime}=100 \mathrm{kPa}$, both experiential and simulated constitutive responses exhibit only hardening. Figures 10a and 10b compare the hardening modulus predicted by the modified MD model with the threshold values for static liquefaction $\left(K_{p}^{\mathrm{liq}}\right)$ and shear banding $\left(K_{p}^{\mathrm{sb}}\right)$. Interestingly, we found that the perfectly incompressible constraint imposed by the trapped pore-fluid may stabilize the material and prevent the formation of shear bands. Furthermore, this comparison also reveals that static liquefaction may occur either (i) near the critical state line or (ii) at the peak of the deviatoric stress. The former case is very similar to a critical state in the drained condition in which the hardening modulus, frictional coefficient and plastic dilatancy are very small and lead to materials shearing as a frictional fluid at constant volume and thus make material very sensitive to effective stress perturbation as demonstrated by the high condition number of $C^{\mathrm{ep}}$. In the latter case, which is coined 'undrained instability' in Andrade (2009), the material become unstable and very sensitive to the total stress perturbation due to the singularity of $C^{\text {und }}$. Notice that strain softening occurs when material is between (i) and (ii). As pointed out by Verdugo and Ishihara (1996), the deviatoric stress at the onset of (ii) strongly depends on the initial confining pressure, whereas the onset of (i) does not. One possible explanation is that the stability triggered by peak deviatoric stress is usually associated with non-zero hardening modulus $K_{p}$ and non-zero frictional coefficient $\beta$ as indicated in Figure 10. This makes the onset of (ii) highly dependent of the initial confining pressure.

Using the localization criteria expressed in Equation (14), we confirm that strain softening does not necessarily guarantee strain localization, which is consistent with the experimental observations of sand made by Chu et al. $(1993,1996)$. 

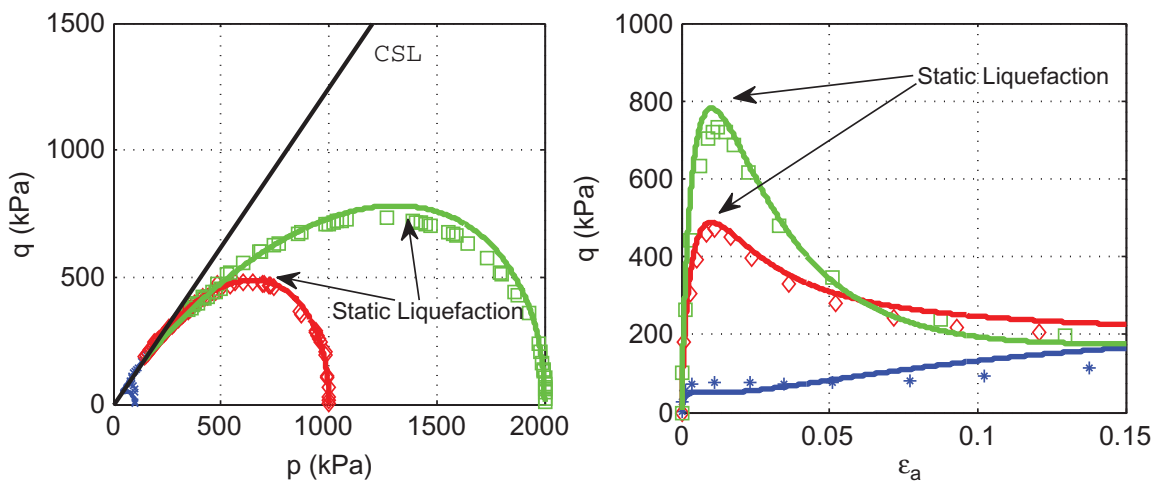

Figure 9. Numerical simulations of undrained triaxial compression tests on Toyoura sand with initial void ratio $=0.907$ : (a) simulated deviatoric stress versus effective hydrostatic pressure and (b) deviatoric stress versus axial strain. The confining pressures of the three simulations are $100 \mathrm{kPa}$ (blue), $1000 \mathrm{kPa}$ (red) and $2000 \mathrm{kPa}$ (green). Experimental data from Verdugo and Ishihara (1996) used to calibrate the material parameters are plotted in dots.
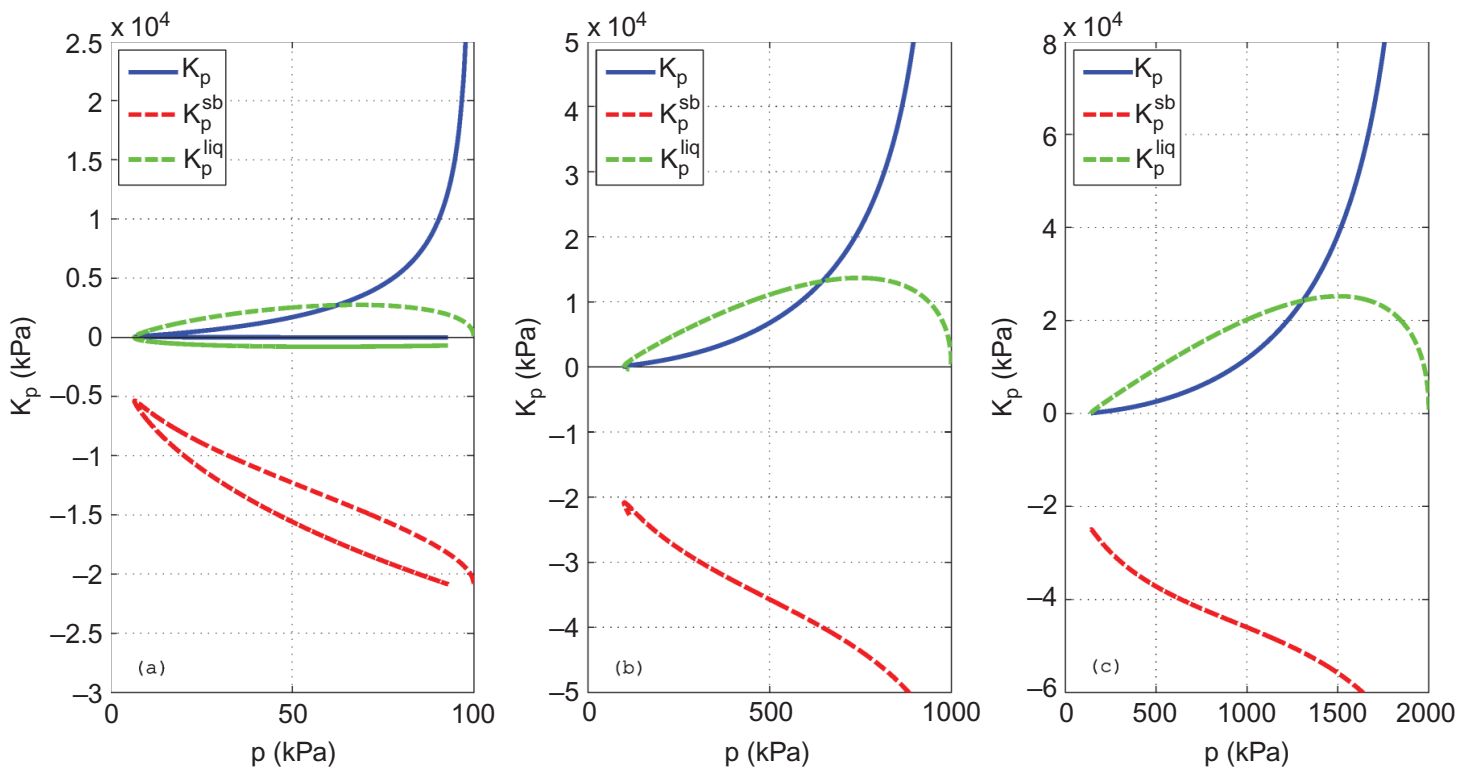

Figure 10. Simulated evolution of hardening modulus $\left(K_{p}\right.$, in blue colour) and the corresponding threshold values for pure shear band ( $K_{p}^{\mathrm{sb}}$, in red colour) and static liquefaction $\left(K_{p}^{\mathrm{liq}}\right.$, in green colour) of Toyoura sand with initial void ratio $=0.907$ and confining pressures equal to (a) $100 \mathrm{kPa}$, (b) $1000 \mathrm{kPa}$, and (c) $2000 \mathrm{kPa}$.
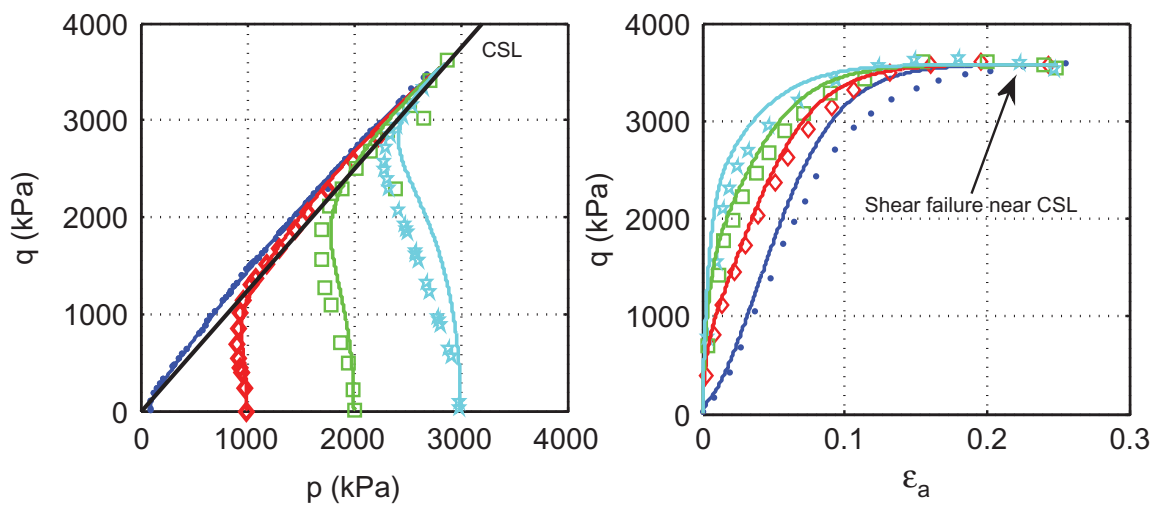

Figure 11. Numerical simulations of undrained triaxial compression tests on Toyoura sand with initial void ratio $=0.735$ : (a) simulated deviatoric stress versus effective hydrostatic pressure and (b) deviatoric stress versus axial strain. The confining pressures of the three simulations are $100 \mathrm{kPa}$ (blue), $1000 \mathrm{kPa}$ (red), $2000 \mathrm{kPa}$ (green) and $3000 \mathrm{kPa}$ (cyan). Experimental data from Verdugo and Ishihara (1996) used to calibrate the material parameters are plotted in dots. 

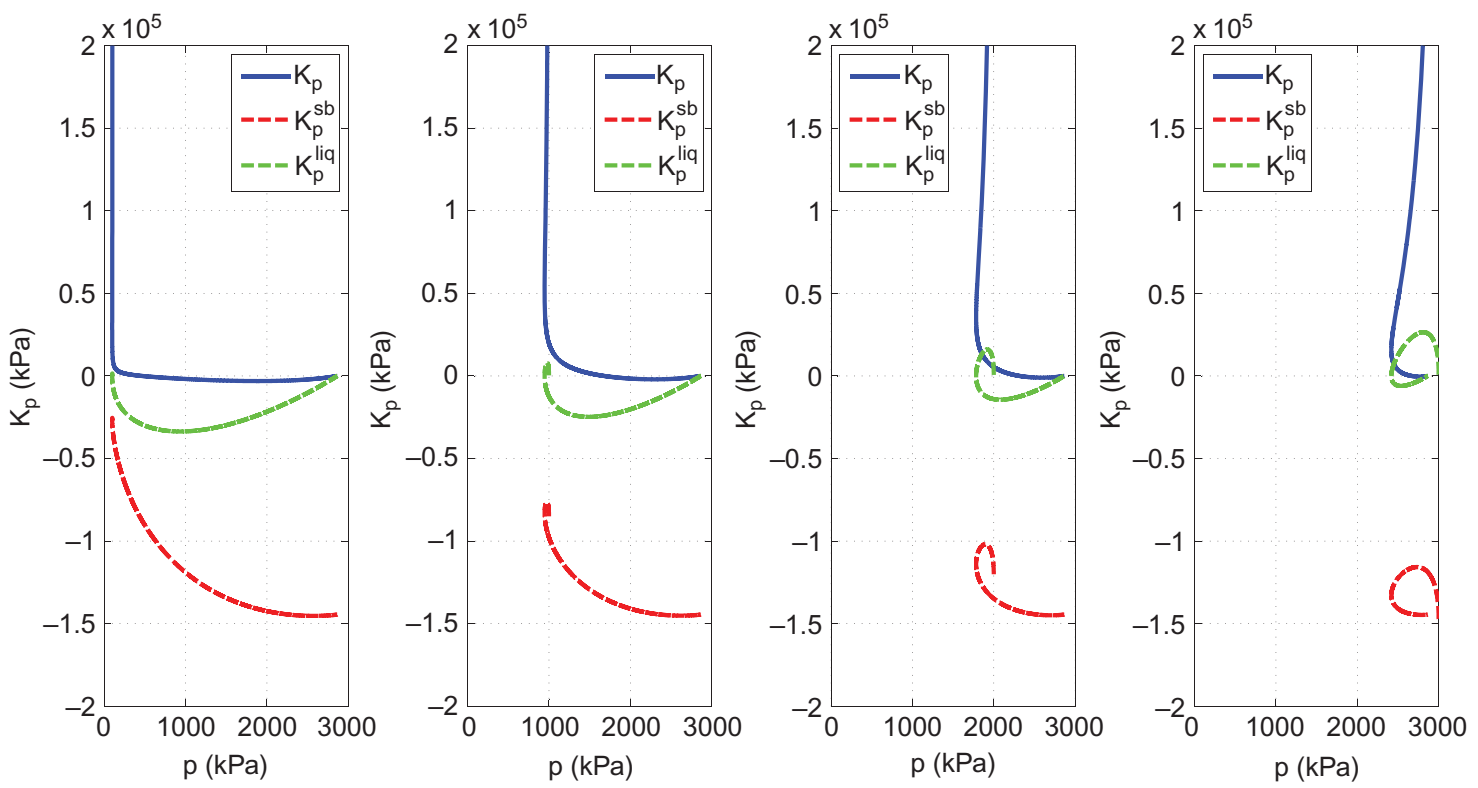

Figure 12. Simulated evolution of hardening modulus ( $K_{p}$, in blue colour) and the corresponding threshold values for pure shear band ( $K_{p}^{\mathrm{sb}}$, in red colour) and static liquefaction $\left(K_{p}^{\mathrm{liq}}\right.$, in green colour) of Toyoura sand with initial void ratio $=0.735$ and confining pressures equal to (a) $100 \mathrm{kPa}$, (b) $1000 \mathrm{kPa}$, (c) $2000 \mathrm{kPa}$, and (d) $3000 \mathrm{kPa}$.

\subsection{Example 4: Stable constitutive response of undrained Toyoura sand in dense state}

We simulate an undrained triaxial compression test on dense Toyoura sand with confining pressures of 100, 1000, 2000 and $3000 \mathrm{kPa}$. The initial void ratio of this simulation is 0.734 . As demonstrated in Figures $11 \mathrm{a}$ and $11 \mathrm{~b}$, the simulated constitutive response matches closely with the experimental data in Verdugo and Ishihara (1996). Unlike loose Toyoura sand, dense Toyoura sand does not exhibit peak deviatoric stress. As a result, shear failures only occur near the critical state line.

Figure 12 compares the generalized hardening modulus with the static liquefaction and shear band threshold values. The simulation result demonstrated in Figure 12 confirms that there is no shear band formed in dense Toyoura sand under undrained conditions. This observation is consistent with the homogeneous deformation modes observed by Verdugo and Ishihara (1996).

\section{Conclusions}

We propose a simple and yet unified method to simulate and predict the onsets of drained collapse, static liquefaction and the formation of deformation bands under drained and undrained conditions. This method is based on bifurcation analyses and a two-invariant critical state plasticity theory. By comparing numerical simulations with experimental data, not only do we show that the instability criteria are capable of delivering predictions consistent with experimental observations, but we also provide a physical interpretation of why the presence of pore-fluid may facilitate or delay instabilities and how contractive/dilatant states affect both the onsets and modes of failure. Using experimental data available in the literature, we compare the simulated and observed constitutive responses as well as the predicted and actual onsets of instability. Our findings confirm that the framework is able to replicate robustly both the constitutive response and the onset of various instability modes observed in experiments.

\section{Acknowledgements}

The author thanks Dr Dariusz Wanatowski of the University of Nottingham for providing experimental data relating to drained triaxial compression tests on Changi sand. Financial support through a graduate fellowship from Northwestern University is gratefully acknowledged. The expert review from the anonymous reviewer is also gratefully acknowledged.

Sandia National Laboratories is a multi-program laboratory managed and operated by the Sandia Corporation, a wholly owned subsidiary of the Lockheed Martin Corporation, for the U.S. Department of Energy's National Nuclear Security Administration under contract DE-AC04-94AL85000.

\section{References}

Andrade, J.E., 2009. A predictive framework for liquefaction instability. Géotechnique, 59 (8), 673-682.

Biot, M.A., 1941. General theory of three-dimensional consolidation. Journal of Applied Physics, 12 (2), 155-161.

Borja, R.I., 2006a. Conditions for instabilities in collapsible solids including volume implosion and compaction banding. Acta Geotechnica, 1 (6), 107-122.

Borja, R.I., 2006b. Conditions for liquefaction instability in fluidsaturated granular soils. Acta Geotechnica, 1 (4), 211-224. 
Borja, R.I., 2007. Localized and diffuse bifurcations in porous rocks undergoing shear localization and cataclastic flow. In: E. Onate and R. Owen, eds. Computational plasticity. Computational methods in applied sciences series.: Springer.

Chu, J., Leroueil, S. and Leong, W.K., 2003. Unstable behavior of sand and its implication for slope instability. Canadian Geotechnical Journal, 40 (5), 873-885.

Chu, J., Lo, R.S.-C. and Lee, I.K., 1993. Instability of granular soils under strain path testing. Journal of Geotechnical Engineering (ASCE), 119 (5), 874-892.

Chu, J., Lo, R.S.-C. and Lee, I.K., 1996. Strain softening and shear band formation of sand in multi-axial testing. Géotechnique, 46 (1), 63-82.

Coussy, O., 2004. Poromechanics. New York: Wiley.

Dafalias, Y.F. and Manzari, M.T., 2004. Simple plasticity sand model accounting for fabric change effects. Journal of Engineering Mechanics, 130 (6), 622-634.

Darve, F., et al., 2007. Bifurcation in granular media: Macroand micro-mechanics approaches. Comptes Rendus Mécanique, 335 (9-10), 496-515.

Hill, R., 1958. A general theory of uniqueness and stability in elasticplastic solids. Journal of the Mechanics and Physics of Solids, 6 (3), 236-249.

Horn, R.A. and Johnson, C.R., 1985. Matrix analysis. New York: Cambridge University Press.

Ikeda, K., Sasaki, H. and Ichimura, T., 2006. Diffuse mode bifurcation of soil causing convention-like shear investigated by grouptheoretic image analysis. Journal of the Mechanics and Physics of Solids, 54 (2), 301-339.

Imam, S.M.R., et al., 2002. Yielding and flow liquefaction of loose sand. Soils and Foundations, 42 (3), 19-31.

Issen, K.A. and Rudnicki, J.W., 2000. Conditions for compaction bands in porous rock. Journal of Geophysical Research, 105 (B9), 21529-21536.

Issen, K.A., 2002. The influence of constitutive models on localization conditions for porous rock. Engineering Fracture Mechanics, 69 (17), 1891-1906.

Jefferies, M.G. and Been, K., 2006. Soil liquefaction: A critical state approach. London: Taylor \& Francis.

Lade, P.V., 1993. Initiation of static liquefaction in the submarine Nerlek berm. Canadian Geotechnical Journal, 30 (6), 895-904.

Lade, P.V. and Pradel, D., 1990. Instability and plastic flow of soils I: Experimental observations. Journal of Engineering Mechanics (ASCE), 116 (11), 2532-2550.

Manzari, M.T. and Dafalias, Y.F., 1997. A two-surface critical plasticity model for sand. Géotechnique, 47 (2), 255-272.
Nur, A. and Byerlee, J.D., 1971. An exact effective stress law for elastic deformation of rock with fluids. Journal of Geophysical Research, 76 (26), 890-990.

Perrin, G. and Leblond, J.B., 1993. Rudnicki and Rice's analysis of strain localization revisited. Journal of Applied Mechanics, 60 (4), 842-847.

Raniecki, B., 1979. Uniqueness criteria in solids with non-associated plastic flow laws at finite deformation. Bulletin of the Polish Academy of Sciences, 27 (8-9), 391-399.

Rice, J.R. and Cleary, M.P., 1976. Some basic stress diffusion solution for fluid-saturated elastic porous media with compressible constituents. Review of Geophysics and Space Physics, 14 (2), 227-241.

Rudnicki, J.W., 2004. Shear and compaction band formation on an elliptic yield cap. Journal of Geophysical Research, 109, B03402. doi:10.1029/2003JB002633

Rudnicki, J.W., 2009. Localization in undrained deformation. In: H.I. Ling, A. Smyth and R. Betti, eds. Poromechanics IV: Proceedings of the fourth Biot conference on poromechanics, including the second Frank L. DiMaggio symposium, Columbia University, New York 8-10 June 2009: Lancaster, Pennsylvania DEStech Publication Inc, 1134-1139.

Rudnicki, J.W. and Rice, J.R., 1975. Conditions for the localization of deformation in pressure-sensitive dilatant materials. Journal of Mechanics and Physics and Solid, 23 (4), 371-394.

Runesson, K., Perić, D. and Sture, S., 1996. Effect of pore fluid compressibility on localization in elastic-plastic porous solids under undrained conditions. International Journal of Solids and Structures, 33 (10), 1501-1518.

Schofield, A.N. and Wroth, C.P., 1968. Critical state soil mechanics. New York: McGraw-Hill.

Terzaghi, K., 1943. Theoretical soil mechanics. New York: Wiley.

Wanatowski, D. and Chu, J., 2007a. Static liquefaction of sand in plane strain. Canadian Geotechnical Journal, 44 (3), 299-313.

Wanatowski, D. and Chu, J., 2007b. Drained behavior of Changi sand in triaxial and plan-strain compression. Geomechanics and Geoengineering, 2 (1), 29-30.

William, K.J., 2002. Constitutive models for engineering materials. In: Encyclopedia of physical science and technology. Vol. 3. 3rd ed. San Diego: Academic Press, 603-633.

Verdugo, R. and Ishihara, K., 1996. The steady state of sandy soils. Soils and Foundations, 36 (2), 81-92.

Zienkiewicz, O.C., et al., 1999. Computational geomechanics with special reference to earthquake engineering. New York: Wiley. 